\title{
Ruthenium-catalyzed formation of pyrazoles or 3-hydroxynitriles from propargyl alcohols and hydrazines
}

\author{
Julia Kaufmann, Elisabeth Jäckel, and Edgar Haak* \\ Institute of Chemistry, Otto von Guericke University Magdeburg, 39106 Magdeburg, Germany \\ Email: edgar.haak@ovgu.de
}

\section{Dedicated to Professor Stephen Hanessian}

Received 01-27-2019

Accepted 03-08-2019

Published on line 03-13-2019

\section{Abstract}

Functionalized pyrazoles are generated from secondary propragyl alcohols and hydrazines in a rutheniumcatalyzed cascade process, consisting of redox isomerization, Michael addition, cyclocondensation and dehydrogenation steps. The same bifunctional catalyst mediates the conversion of tertiary propargyl alcohols with hydrazine to 3-hydroxynitriles via anti-Markovnikov hydroamination followed by elimination of ammonia.

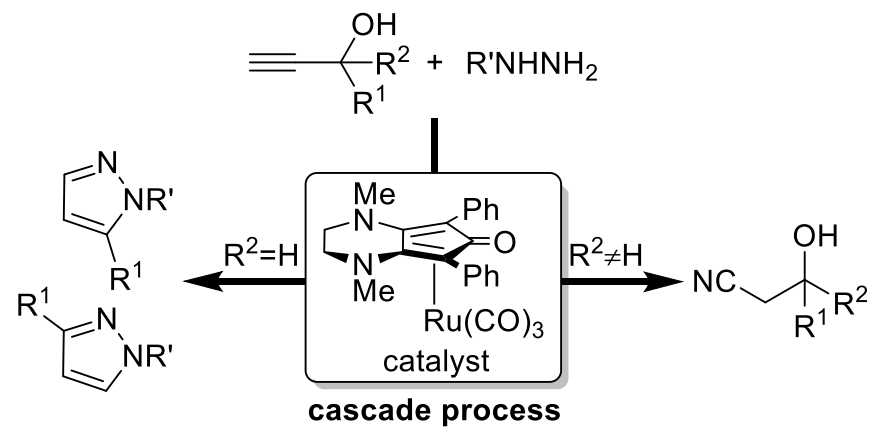

Keywords: Cascade reactions, homogeneous catalysis, propargyl alcohols, pyrazoles, ruthenium 


\section{Introduction}

The pyrazole nucleus represents a pharmacologically privileged substructure that occurs in numerous pharmaceutical and agrochemical agents. ${ }^{1-3}$ Various types of pharmacological properties, such as antibacterial, antifungal, anticancer, antidepressant, antiinflammatory, anti-tuberculosis, antioxidant as well as antiviral activity have been described for pyrazole derivatives. ${ }^{1,2}$ Consequently, a variety of efficient and versatile synthetic methods have been developed to assemble this structural motif. ${ }^{2-11}$ The 1,3-dipolar cycloaddition of diazoalkanes or nitrile imines with olefins and alkynes is limited in terms of the accessibility of the 1,3dipoles. $^{2-4}$ The more frequently applied classical cyclocondensation of hydrazines with 1,3-dicarbonyl compounds (Knorr synthesis) or acetylenic ketones provides a rapid approach to obtain substituted pyrazoles. $^{2-7}$ Alternatively, the more readily available enones can be used, but this requires an additional oxidation step. ${ }^{2-4,8-11}$ In this context, ruthenium catalysis is often utilized and various modes of oxidative catalytic activity are applied, including oxidative C-N coupling, ${ }^{9}$ hydrogen transfer, ${ }^{10}$ and visible light photoredox catalysis. ${ }^{11}$

Some years ago, we discovered that bifunctional ruthenium cyclopentadienone complexes such as catalyst 1 and derivatives thereof bring about various selective transformations of propargyl alcohols with diverse nucleophiles. Secondary propargyl alcohols are converted in a redox-isomerization / Michael addition cascade. ${ }^{12-16}$ Saturated tertiary substrates generate diverse addition or substitution products, ${ }^{13,15,20}$ whereas tertiary 1-alkenyl propargyl alcohols undergo an allylation/cycloisomerization cascade process. . $^{13,14,16-20}$ Herein, we report on a mechanistically related formation of pyrazoles from secondary propargyl alcohols and hydrazines as well as the generation of 3-hydroxynitriles from tertiary substrates applying similar reaction conditions (Scheme 1).

Classical condensation:

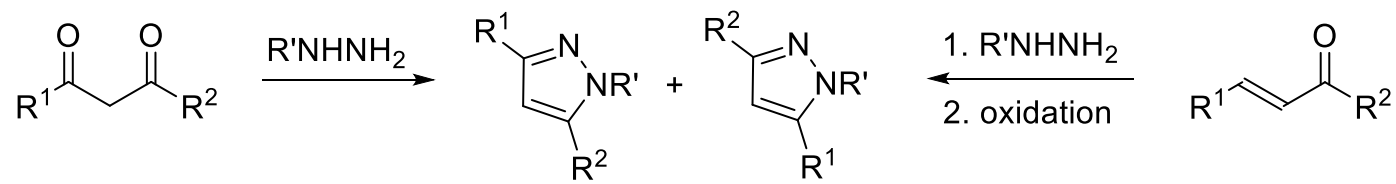

This work:
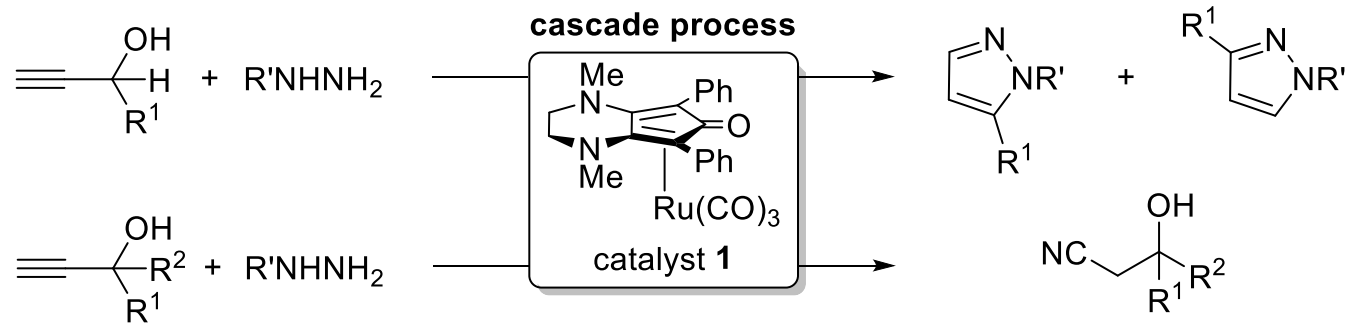

Scheme 1. Conventional pyrazole synthesis and ruthenium catalyzed cascade conversions.

\section{Results and Discussion}

The ruthenium-catalyzed conversion of secondary propargyl alcohols $\mathbf{2 a - j}$ with hydrazine hydrate led to the formation of various pyrazoles $\mathbf{3}$. The cascade reaction proceeds via cycloisomerization, Michael addition, 
cyclocondensation and dehydrogenation steps. Almost quantitative product formation is observed from substrates $\mathbf{2 b - 2 e}$ containing aryl or sterically demanding alkenyl substituents including terpene-derived starting materials. Alkyl-substituted substrates $\mathbf{2 a}$ and $\mathbf{2 f}$ led to small amounts of 3-hydroxynitriles $\mathbf{4}$ as byproducts, whereas the hydroamination side product (5) is formed solely from the sterically simplest derivative $\mathbf{2 a}$. Compounds $\mathbf{2} \mathbf{g}-\mathbf{2} \mathbf{j}$ containing sterically less demanding alkenyl side chains generate regioisomeric dihydro pyrazoles 6 as the minor components (Scheme 2). No conversion of the propargyl alcohols is observed in absence of the ruthenium catalyst.
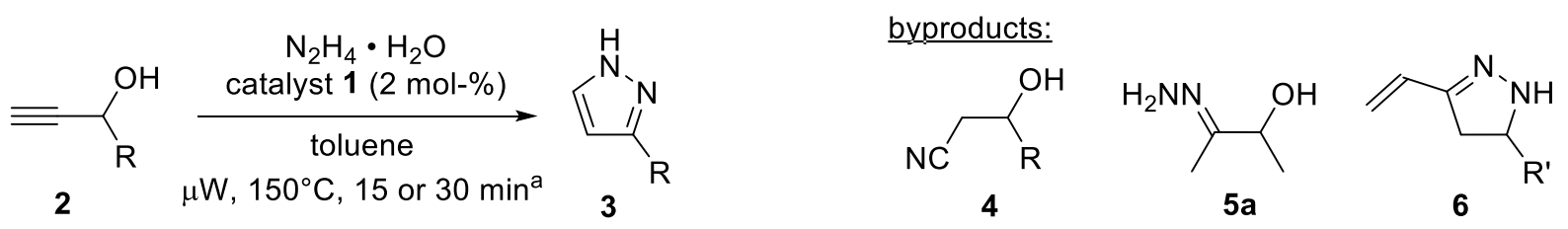

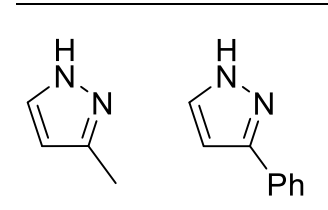

$3 a 32 \%$

(+ 4a $30 \%$ 3b $96 \% \quad$ 3c $98 \%$

+5 a $25 \%$ )

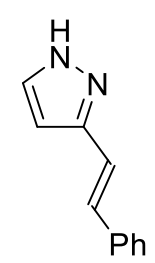

3c $98 \%$ 3d $99 \%$

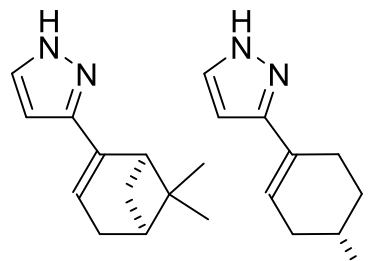

3e $99 \%$
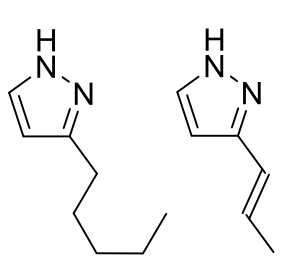

3 g $37 \%$

$(+4 f 10 \%)(+6 g 41 \%)$

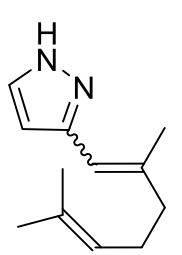

3h $84 \%$

$(+6 h 8 \%)$
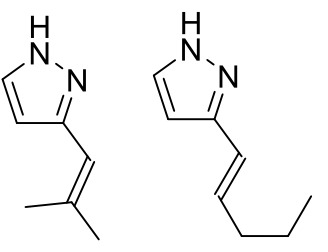

$3 \mathbf{i} 71 \% \quad 3 \mathbf{j} 52 \%$

$(+6 \mathbf{i} 11 \%)(+6 \mathbf{j} 28 \%)$

${ }^{a}$ Reaction time: 15 min for $\mathbf{2 a - e , ~} \mathbf{2 g}, \mathbf{2} \mathbf{j}$; 30 min for $\mathbf{2 f}, \mathbf{2 h}, \mathbf{2} \mathbf{i}$.

Scheme 2. Ruthenium catalyzed synthesis of pyrazoles from secondary propargyl alcohols and hydrazine.

If phenylhydrazine is applied as the nucleophilic component, regioisomeric pyrazoles $\mathbf{7}$ and $\mathbf{8}$ are obtained selectively. Byproduct formation is observed only using the sterically simple substrate $\mathbf{2 a}$. Substrates $\mathbf{2 b}$ and $\mathbf{2} \mathbf{i}$ react slowly and some unreacted educt was recovered. Substrate $\mathbf{2 a}$ forms isomer $\mathbf{7}$ as the major compound, whereas unsaturated side chains led to pyrazoles $\mathbf{8}$ as the major products (Scheme 3). 

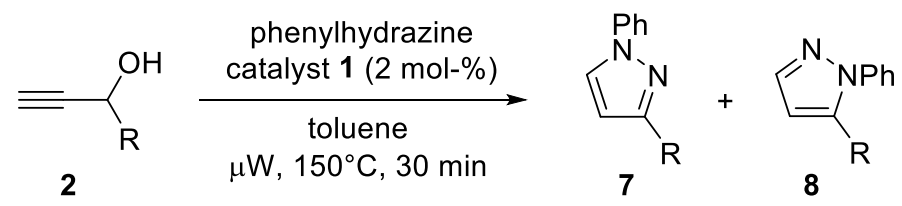

byproduct:

PhHNN

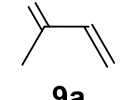

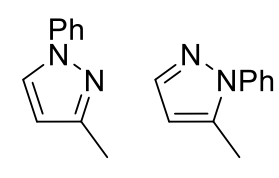

$7 a 40 \%+8 a 9 \%$ $(+9 a 29 \%)$

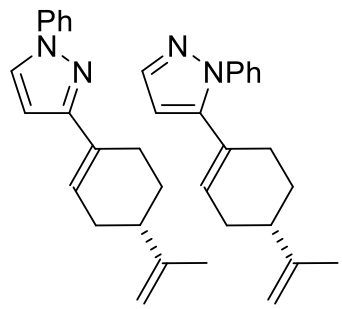

7 e $31 \%+8$ e $62 \%$

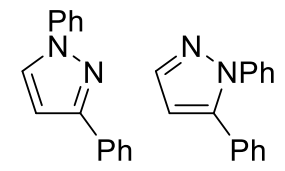

7b $15 \%+8$ b $39 \%$

(+ 2b 22\%)

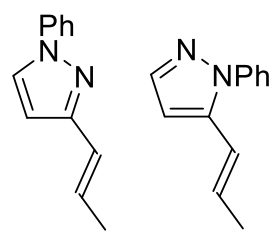

7 g $25 \%+8 g 62 \%$

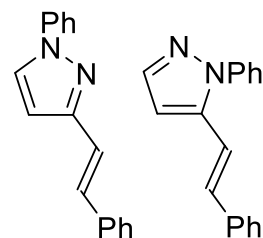

7c $38 \%+8$ c $56 \%$

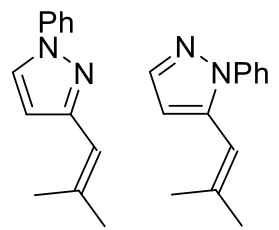

$7 \mathbf{i}<5 \%+8 \mathbf{i} 59 \%$ $(+2 \mathbf{i} 28 \%)$

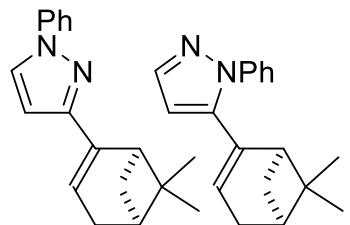

$7 d 28 \%+8 d 60 \%$

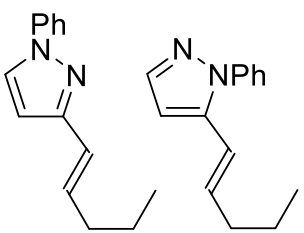

$7 \mathbf{j} 21 \%+8 \mathbf{j} 59 \%$

Scheme 3. Formation of regioisomeric pyrazoles from secondary propargyl alcohols and phenyl hydrazine.

The formation of byproduct $\mathbf{4}$ from hydrazine hydrate and alkyl-substituted propargyl alcohols $\mathbf{2 a}$ and $\mathbf{2 f}$ led us to investigate the formation of similar 3-hydroxynitriles $\mathbf{4}$ from tertiary propargyl alcohols $\mathbf{2 k - 0}$. Almost quantitative product formation is observed from alkyl-substituted substrates $\mathbf{2 k}$ and $\mathbf{2}$. Propargyl alcohols $\mathbf{2 n}$ and $\mathbf{2 0}$ are converted much more slowly and some unreacted educt $\mathbf{2}$ was recovered. The hydroamination side product (5) is formed solely from the sterically simple derivative $\mathbf{2 m}$, whereas $\mathrm{N}$-aminopyrroles $\mathbf{1 0}$ are generated as byproducts from substrates $\mathbf{2 m - 2 0}$ containing alkenyl side chains (Scheme 4 ). The propargyl alcohols are not converted in absence of the ruthenium catalyst. Only trace amounts of hydroxynitriles $\mathbf{4}$ are generated from substituted hydrazines (e.g. phenylhydrazine, tert.-butylhydrazine, N,N-dimethylhydrazine) and propargyl alcohols $\mathbf{2}$ applying similar reaction conditions. A related rhodium catalyzed conversion of tertiary propargyl alcohols with tert.-butylhydrazine was previously reported by Fukumoto et al. ${ }^{21}$
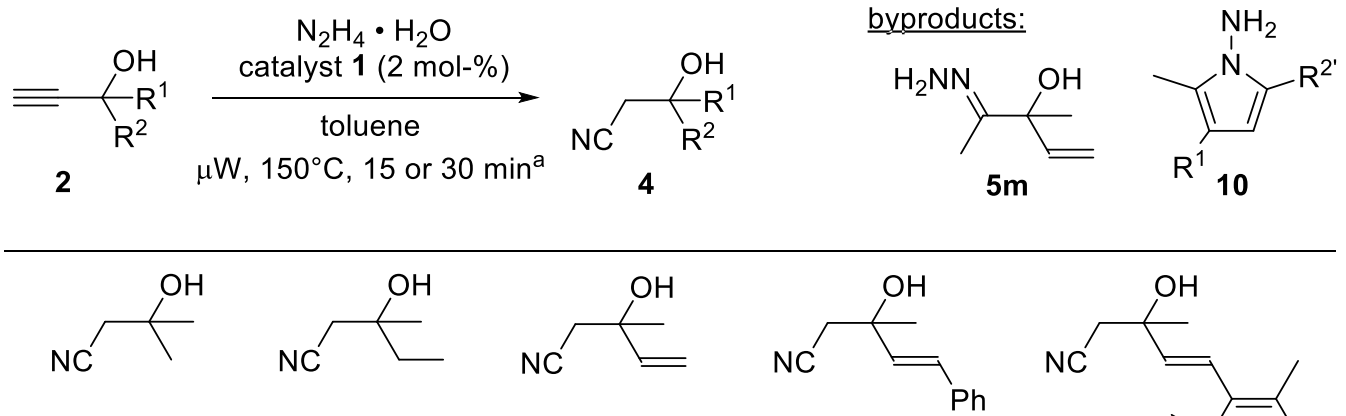

4k $91 \%$
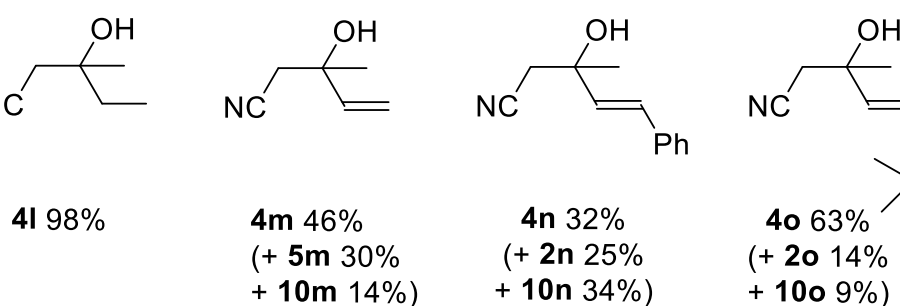

${ }^{a}$ Reaction time: 15 min for $\mathbf{2 k - m ;} 30$ min for $\mathbf{2 n}, \mathbf{2 0}$.

Scheme 4. Ruthenium catalyzed synthesis of 3-hydroxynitriles from tertiary propargyl alcohols and hydrazine. 
In accordance with our previously reported results, ${ }^{12-20}$ substrate activation occurs by initial formation of the chelated $\pi$-complex I and its subsequent conversion into alkynyl species II. Secondary substrates are further converted to alkenyl complex III by a 1,2-hydrogen shift. Regioselective nucleophilic attack at the metalated carbon followed by a cyclocondensation step leads to alkyl complex $\mathbf{V}$ that liberates the pyrazole

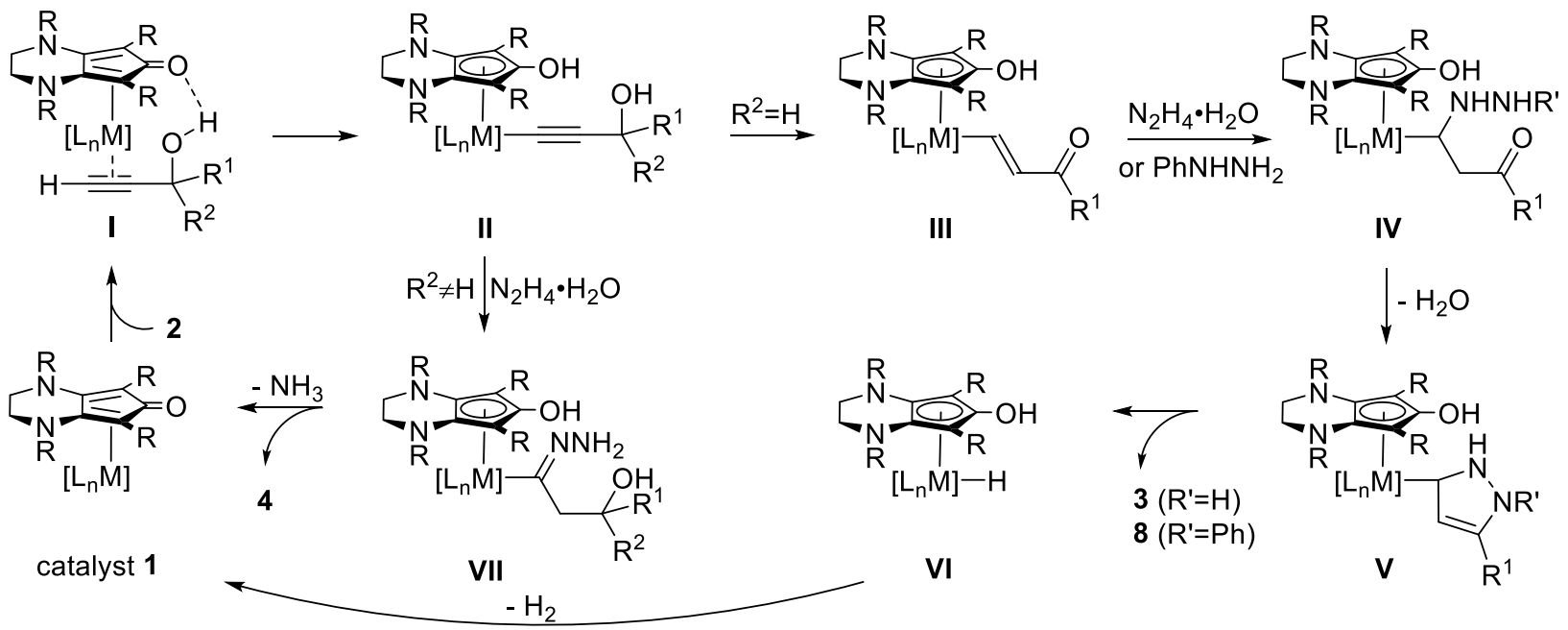

Scheme 5. Proposed mechanisms for the catalytic transformations.

$(3,8)$ via $\beta$-hydride elimination. Regeneration of the active catalyst occurs by elimination of hydrogen from the ruthenium hydride VI. If tertiary substrates are applied, the nucleophile already attacks the alkynyl complex II, also at the metalated carbon atom. Elimination of ammonia from hydrazone species VII regenerates the active catalyst and liberates the 3-hydroxynitrile (4). Formation of regioisomeric $\mathrm{N}$-substituted pyrazoles 7 occurs presumably by initial hydrazone formation from intermediate III. Side products $\mathbf{5}$ and $\mathbf{9}$ are formed via nucleophilic attack at the internal carbon atom of alkynyl complex II, if sterically simple substrates (2a, 2m) are applied. Side products $\mathbf{6}$ and $\mathbf{1 0}$ result from initial nucleophilic attack at the non-metalated double bond, if alkenyl substituted substrates are applied (Scheme 5).

\section{Conclusions}

We present ruthenium-catalyzed one pot transformations of readily available propargyl alcohols with hydrazines to yield functionalized pyrazoles or 3-hydroxynitriles, selectively. Aldehyde-derived secondary substrates are converted into pyrazoles in a cascade process, consisting of redox isomerization, Michael addition, cyclocondensation and dehydrogenation steps. Ketone-derived tertiary substrates form 3-hydroxynitriles via anti-Markovnikov hydroamination followed by elimination of ammonia. The processes are catalyzed by a bifunctional ruthenium complex containing a redox-coupled cyclopentadienone ligand. Such transition-metal catalyzed cascade reactions are of special interest in the context of drug discovery. The incorporation of multiple structural transformations in one process carried out in one pot increases synthetic efficiency significantly and facilitates the rapid generation of various structural analogues utilizing readily available starting materials. 


\section{Experimental Section}

\section{General Information}

All reactions were carried out in a dry atmosphere under argon. The chemicals used were dried and purified according to common procedures. Products were purified by flash chromatography on silica and identified by spectroscopic analysis ( ${ }^{1} \mathrm{H}$ NMR, ${ }^{13} \mathrm{C}$ NMR, IR, MS, HRMS). Multiplicity was determined by DEPT spectra for all compounds. Infrared spectra were obtained with a Perkin-Elmer FT-IR 2000. NMR spectra were recorded with a Bruker DPX 400 or a Bruker Avance 600 spectrometer. MS and HRMS data were obtained with a Finnigan MAT 95 or a Waters Acquity UPLC-MS H-Class. Reactions using microwave irradiation were performed in a sealed tube with an Anton-Paar Monowave 300 reactor. Catalyst 1 has been prepared previously, ${ }^{12}$ and its crystal structure has been solved. ${ }^{13}$

\section{General catalytic procedure}

Catalyst 1 (0.02 mmol) was dissolved in toluene $(2 \mathrm{~mL})$ and the propargyl alcohol (1 mmol) and hydrazine hydrate $(2 \mathrm{mmol})$ or phenylhydrazine $(1 \mathrm{mmol})$ were subsequently added. The mixture was heated to $150{ }^{\circ} \mathrm{C}$ for 15-30 minutes using microwave irradiation. Evaporation of the solvent and flash chromatography on silica (gradient: pentane/ethyl acetate) furnished the purified products as colorless or yellow oils or foams.

Spectral data of compounds $3 a,{ }^{22} 3 b,{ }^{23} 4 a,{ }^{24} 4 f,{ }^{25} 4 k^{26} 4{ }^{26},{ }^{27} 4 m,{ }^{28} 4 n,{ }^{28} 4 o,{ }^{29} 7 a,{ }^{30} 7 b,{ }^{31} 8 a,{ }^{30}$ and $8 b^{32}$ are in agreement with the data previously reported by others.

(E)-3-Styryl-1H-pyrazole (3c). ${ }^{1} \mathrm{H}$ NMR $\left(600 \mathrm{MHz}, \mathrm{CDCl}_{3}\right): \delta 7.53$ (d, J $\left.2.2 \mathrm{~Hz}, 1 \mathrm{H}\right), 7.47$ (t, J $\left.7.6 \mathrm{~Hz}, 2 \mathrm{H}\right), 7.33(\mathrm{t}, J$ $7.4 \mathrm{~Hz}, 2 \mathrm{H}), 7.24(\mathrm{tt}, J 7.3,1.3 \mathrm{~Hz}, 1 \mathrm{H}), 7.12(\mathrm{~d}, J 16.6 \mathrm{~Hz}, 1 \mathrm{H}), 7.08(\mathrm{~d}, J 16.6 \mathrm{~Hz}, 1 \mathrm{H}), 6.47(\mathrm{~d}, J 2.2 \mathrm{~Hz}, 1 \mathrm{H}) .{ }^{13} \mathrm{C}$ $\operatorname{NMR}\left(150 \mathrm{MHz}, \mathrm{CDCl}_{3}\right): \delta 146.7$ (br, C), 136.8 (C, C2), $133.9(\mathrm{br}, \mathrm{CH}), 130.5(\mathrm{CH}), 128.7(2 \mathrm{CH}), 127.9(\mathrm{CH}), 126.4$ (2CH), $118.2(\mathrm{br}, \mathrm{CH}), 102.6(\mathrm{CH}) . \mathrm{IR}(\mathrm{ATR}): \mathrm{v}_{\max }$ 3181, 3035, 2956, 1672, 1467, 1369, 1205, 1098, 1048, 966, 768, 752, $694 \mathrm{~cm}^{-1}$. MS (EI): m/z (\%): 170 [M] (86), 169 (100), 142 (22), 84 (50). HRMS (EI): m/z calcd. for $\mathrm{C}_{11} \mathrm{H}_{10} \mathrm{~N}_{2}[\mathrm{M}]^{+}:$170.0839; Found: 170.0839.

3-((1R,5S)-6,6-Dimethylbicyclo[3.1.1]hept-2-en-2-yl)-1H-pyrazole (3d). ${ }^{1} \mathrm{H}-\mathrm{NMR}\left(600 \mathrm{MHz}, \mathrm{CDCl}_{3}\right): \delta 7.50$ (d, J $2.1 \mathrm{~Hz}, 1 \mathrm{H}), 6.27$ (d, J $2.1 \mathrm{~Hz}, 1 \mathrm{H}), 6.04(\mathrm{tt}, J 3.2,1.5 \mathrm{~Hz}, 1 \mathrm{H}), 2.74(\mathrm{td}, J 5.6,1.5 \mathrm{~Hz}, 1 \mathrm{H}), 2.50$ (dt, J 8.7, $5.6 \mathrm{~Hz}$, 1H), $2.46(\mathrm{dt}, J 18.6,3.2 \mathrm{~Hz}, 1 \mathrm{H}), 2.39(\mathrm{dt}, J 18.6,3.0 \mathrm{~Hz}, 1 \mathrm{H}), 2.20-2.15(\mathrm{~m}, 1 \mathrm{H}), 1.36(\mathrm{~s}, 3 \mathrm{H}), 1.29(\mathrm{~d}, J 8.7 \mathrm{~Hz}$, $1 \mathrm{H}), 0.87(\mathrm{~s}, 3 \mathrm{H}) .{ }^{13} \mathrm{C}-\mathrm{NMR}\left(150 \mathrm{MHz}, \mathrm{CDCl}_{3}\right): \delta 147.2(\mathrm{br}, \mathrm{C}), 138.3(\mathrm{C}), 135.5(\mathrm{br}, \mathrm{CH}), 120.1(\mathrm{CH}), 101.5(\mathrm{CH})$, $44.0(\mathrm{CH}), 40.8(\mathrm{CH}), 38.1(\mathrm{C}), 31.8\left(\mathrm{CH}_{2}\right), 31.5(\mathrm{CH}), 26.3\left(\mathrm{CH}_{3}\right), 21.0\left(\mathrm{CH}_{3}\right) . \mathrm{IR}: v_{\max }$ 3215, 2917, 2872, 1666, 1465, 1365, 1195, 1102, 1050, 909, 886, 765, 730, 647. MS (ESI): m/z (\%): $188[\mathrm{M}]^{+}$(76), 173 (74), 146 (31),

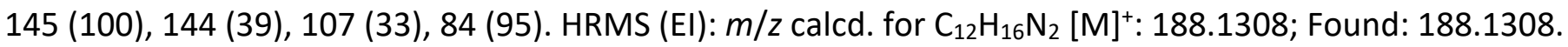

(S)-3-[4-(Prop-1-en-2-yl)cyclohex-1-en-1-yl]-1H-pyrazole (3e). ${ }^{1} \mathrm{H}-\mathrm{NMR}\left(600 \mathrm{MHz}, \mathrm{CDCl}_{3}\right): \delta 7.52(\mathrm{~d}, \mathrm{~J} 2.1 \mathrm{~Hz}$, 1H), $6.32(\mathrm{br} \mathrm{s}, 1 \mathrm{H}), 6.31(\mathrm{~d}, J 2.1 \mathrm{~Hz}, 1 \mathrm{H}), 4.80-4.77(\mathrm{~m}, 2 \mathrm{H}), 2.65-2.58(\mathrm{~m}, 1 \mathrm{H}), 2.51-2.41(\mathrm{~m}, 1 \mathrm{H}), 2.37-2.22(\mathrm{~m}$, $2 \mathrm{H}), 2.18-2.09(\mathrm{~m}, 1 \mathrm{H}), 2.00-1.94(\mathrm{~m}, 1 \mathrm{H}), 1.80(\mathrm{~s}, 3 \mathrm{H}), 1.61$ (dtd, J 12.7, 11.3, $5.5 \mathrm{~Hz}, 1 \mathrm{H}) .{ }^{13} \mathrm{C}-\mathrm{NMR}(150 \mathrm{MHz}$, $\mathrm{CDCl}_{3}$ ): $\delta 149.5(\mathrm{C}), 148.4(\mathrm{br}, \mathrm{C}), 134.9(\mathrm{br}, \mathrm{CH}), 128.0(\mathrm{C}), 124.3(\mathrm{CH}), 108.9\left(\mathrm{CH}_{2}\right), 101.2(\mathrm{CH}), 40.8(\mathrm{CH}), 30.9$ $\left(\mathrm{CH}_{2}\right), 27.6\left(\mathrm{CH}_{2}\right), 26.8\left(\mathrm{CH}_{2}\right), 20.9\left(\mathrm{CH}_{3}\right) . \mathrm{IR}: \mathrm{v}_{\max }$ 3184, 3082, 2920, 1644, 1451, 1434, 1373, 1284, 1198, 1107, 1050, 934, 885, 831, $761 \mathrm{~cm}^{-1}$. MS (EI): m/z (\%): 188 [M] (67), 147 (61), 120 (77), 119 (100), 93 (59), 81 (33). HRMS (EI): $m / z$ calcd. for $\mathrm{C}_{12} \mathrm{H}_{16} \mathrm{~N}_{2}[\mathrm{M}]^{+}:$: 188.1308; Found 188.1308 .

3-Pentyl-1H-pyrazole (3f). ${ }^{1} \mathrm{H}$ NMR $\left(600 \mathrm{MHz}^{\mathrm{C} C \mathrm{CD}_{3}}\right.$ ): $\delta 7.50$ (d, J $\left.1.9 \mathrm{~Hz}, 1 \mathrm{H}\right), 6.09$ (d, J $\left.1.9 \mathrm{~Hz}, 1 \mathrm{H}\right), 2.69$ (t, J 7.8 $\mathrm{Hz}, 2 \mathrm{H}), 1.68$ (br quint, J $7.6 \mathrm{~Hz}, 2 \mathrm{H}), 1.36-1.34(\mathrm{~m}, 4 \mathrm{H}), 0.91(\mathrm{t}, J 7.0 \mathrm{~Hz}, 1 \mathrm{H}) .{ }^{13} \mathrm{C} \mathrm{NMR}\left(150 \mathrm{MHz}^{\mathrm{C}} \mathrm{CDCl}\right): \delta$ 147.9 (br, C), $135.1(\mathrm{br}, \mathrm{CH}), 103.4(\mathrm{CH}), 31.6\left(\mathrm{CH}_{2}\right), 29.2\left(\mathrm{CH}_{2}\right), 26.7\left(\mathrm{CH}_{2}\right), 22.5\left(\mathrm{CH}_{2}\right), 14.1\left(\mathrm{CH}_{3}\right)$. IR $(\mathrm{ATR}): V_{\max }$ $3194,2955,2928,2867,2859,1686,1466,1434,1377,1295,1202,1111,1050,933,827,757 \mathrm{~cm}^{-1}$. MS (EI): 
m/z (\%): 138 [M]+ (82), 109 (59), 96 (79), 95 (91), 83 (82), 82 (100), 81 (92). HRMS (EI): m/z calcd. for $\mathrm{C}_{8} \mathrm{H}_{14} \mathrm{~N}_{2}$ $[\mathrm{M}]^{+}:$138.1152; Found: 138.1152 .

(E)-3-(Prop-1-en-1-yl)-1H-pyrazole (3g). ${ }^{1} \mathrm{H}$ NMR (600 MHz, CDCl 3 ): $\delta 7.50$ (d, J $\left.2.1 \mathrm{~Hz}, 1 \mathrm{H}\right), 6.40$ (dq, J 16.0, 1.7 $\mathrm{Hz}, 1 \mathrm{H}), 6.30(\mathrm{~d}, J 2.1 \mathrm{~Hz}, 1 \mathrm{H}), 6.24(\mathrm{dq}, J$ 15.9, $6.6 \mathrm{~Hz}, 1 \mathrm{H}), 1.89$ (dd, J 6.6, $1.6 \mathrm{~Hz}, 3 \mathrm{H}) .{ }^{13} \mathrm{C} \mathrm{NMR}(150 \mathrm{MHz}$, $\left.\mathrm{CDCl}_{3}\right): \delta 146.6(\mathrm{br}, \mathrm{C}), 134.5(\mathrm{br}, \mathrm{CH}), 128.3(\mathrm{CH}), 120.9(\mathrm{CH}), 102.0(\mathrm{CH}), 18.6\left(\mathrm{CH}_{3}\right) . \mathrm{HRMS}(\mathrm{El}): \mathrm{m} / \mathrm{z}$ calcd. for $\mathrm{C}_{6} \mathrm{H}_{8} \mathrm{~N}_{2}[\mathrm{M}]^{+}:$108.0682; Found: 108.0683.

3-(2,6-Dimethylhepta-1,5-dien-1-yl)-1H-pyrazole (3h). E-Isomer: ${ }^{1} \mathrm{H} \mathrm{NMR}\left(600 \mathrm{MHz}, \mathrm{CDCl}_{3}\right): \delta 7.56(\mathrm{~d}, \mathrm{~J} 2.0 \mathrm{~Hz}$, $1 \mathrm{H}), 6.29(\mathrm{~d}, J 2.0 \mathrm{~Hz}, 1 \mathrm{H}), 6.22(\mathrm{br} \mathrm{s}, 1 \mathrm{H}), 5.16-5.13(\mathrm{~m}, 1 \mathrm{H}), 2.21-2.17(\mathrm{~m}, 4 \mathrm{H}), 1.96(\mathrm{~d}, J 1.2 \mathrm{~Hz}, 3 \mathrm{H}), 1.70(\mathrm{br} \mathrm{s}$, 3H), 1.63 (br s, 3H). ${ }^{13} \mathrm{C} \mathrm{NMR} \mathrm{(150} \mathrm{MHz,} \mathrm{CDCl}$ ): $\delta 143.9$ (br, C), $141.6(\mathrm{C}), 136.1$ (br, CH), $132.1(\mathrm{C}), 123.7(\mathrm{CH})$, $113.9(\mathrm{CH}), 104.3(\mathrm{CH}), 40.9\left(\mathrm{CH}_{2}\right), 26.7\left(\mathrm{CH}_{2}\right), 25.8\left(\mathrm{CH}_{3}\right), 18.6\left(\mathrm{CH}_{3}\right), 17.8\left(\mathrm{CH}_{3}\right)$. IR (ATR): $v_{\max } 3186,3110$, 2967, 2855, 1660, 1447, 1376, 1275, 1206, 1169, 1107, 1050, 934, 868, $756 \mathrm{~cm}^{-1}$. HRMS (El): $\mathrm{m} / \mathrm{z}$ calcd. for $\mathrm{C}_{12} \mathrm{H}_{18} \mathrm{~N}_{2}$ : 190.1465 [M] ; found: 190.1465. Z-Isomer: ${ }^{1} \mathrm{H}$ NMR $\left(600 \mathrm{MHz}, \mathrm{CDCl}_{3}\right): \delta 7.54(\mathrm{~d}, J 1.8 \mathrm{~Hz}, 1 \mathrm{H}), 6.24$ (d, J $1.8 \mathrm{~Hz}, 1 \mathrm{H}), 6.17$ (br s, 1H), 5.19-5.17 (m, 1H), 2.35 (dd, J 8.3, 7.4 Hz, 2H), 2.22-2.19 (m, $2 \mathrm{H}), 1.92(\mathrm{~d}, J 1.1$ $\mathrm{Hz}, 3 \mathrm{H}), 1.71$ (br s, 3H), 1.64 (br s, 3H). $\left.{ }^{13} \mathrm{C} \mathrm{NMR} \mathrm{(150} \mathrm{MHz,} \mathrm{CDCl}\right): \delta 143.3(\mathrm{br}, \mathrm{C}), 142.8(\mathrm{C}), 137.2(\mathrm{br}, \mathrm{CH})$, $132.9(\mathrm{C}), 124.1(\mathrm{CH}), 114.5(\mathrm{CH}), 104.4(\mathrm{CH}), 33.9\left(\mathrm{CH}_{2}\right), 26.4\left(\mathrm{CH}_{2}\right), 26.1\left(\mathrm{CH}_{3}\right), 24.8\left(\mathrm{CH}_{3}\right), 18.1\left(\mathrm{CH}_{3}\right) . \mathrm{HRMS}$ (EI): $m / z$ calcd. for $\mathrm{C}_{12} \mathrm{H}_{18} \mathrm{~N}_{2}: 190.1465$ [M] $]^{+}$found: 190.1466.

3-(2-Methylprop-1-en-1-yl)-1H-pyrazole (3i). ${ }^{1} \mathrm{H}$ NMR (600 MHz, CDCl 3 ): $\delta 7.55$ (d, J $\left.1.8 \mathrm{~Hz}, 1 \mathrm{H}\right), 6.27$ (d, J 1.8 $\mathrm{Hz}, 1 \mathrm{H}), 6.20$ (br s, $1 \mathrm{H}), 1.96$ (d, J $0.7 \mathrm{~Hz}, 3 \mathrm{H}), 1.93$ (d, J $1.1 \mathrm{~Hz}, 3 \mathrm{H}) .{ }^{13} \mathrm{C} \mathrm{NMR}\left(150 \mathrm{MHz}, \mathrm{CDCl}_{3}\right): \delta 143.9(\mathrm{br}, \mathrm{C})$, $138.4(\mathrm{C}), 136.3(\mathrm{br}, \mathrm{CH}), 114.2(\mathrm{CH}), 104.3(\mathrm{CH}), 27.1\left(\mathrm{CH}_{3}\right), 20.1\left(\mathrm{CH}_{3}\right)$. IR (ATR): $v_{\max } 3179,3101,2927,1665$, 1462, 1441, 1377, 1280, 1207, 1109, 1051, 934, 839, $761 \mathrm{~cm}^{-1} . \mathrm{MS}$ (EI): m/z (\%): 122 [M] ${ }^{+}$(100), 107 (27), 94 (20); HRMS (EI): $m / z$ calcd. for $\mathrm{C}_{7} \mathrm{H}_{10} \mathrm{~N}_{2}: 122.0839$ [M] ${ }^{+}$; Found: 122.0838.

(E)-3-(Pent-1-en-1-yl)-1H-pyrazole (3j). ${ }^{1} \mathrm{H}$ NMR (600 MHz, CDCl $): \delta 7.51$ (d, J $\left.2.1 \mathrm{~Hz}, 1 \mathrm{H}\right), 6.40$ (dt, J 16.0, 1.3 $\mathrm{Hz}, 1 \mathrm{H}), 6.32(\mathrm{~d}, J 2.1 \mathrm{~Hz}, 1 \mathrm{H}), 6.25$ (dt, J 16.0, $6.9 \mathrm{~Hz}, 1 \mathrm{H}), 2.19$ (qd, J 7.1, 1.3 Hz, 2H), 1.50 (sext, J 7.4 Hz, 2H), $0.96(\mathrm{t}, \mathrm{J} 7.4 \mathrm{~Hz}, 3 \mathrm{H}) .{ }^{13} \mathrm{C} \mathrm{NMR}\left(150 \mathrm{MHz}, \mathrm{CDCl}_{3}\right): \delta 146.5(\mathrm{br}, \mathrm{C}), 134.6(\mathrm{br}, \mathrm{CH}), 133.4(\mathrm{CH}), 119.7(\mathrm{CH}), 102.0$ (CH), $35.1\left(\mathrm{CH}_{2}\right), 22.4\left(\mathrm{CH}_{2}\right), 13.8\left(\mathrm{CH}_{3}\right)$. IR (ATR): $v_{\max }$ 3175, 3100, 2958, 2928, 2871, 1662, 1462, 1436, 1377, 1296, 1200, 1102, 1052, 962, 934, $759 \mathrm{~cm}^{-1} . \mathrm{MS}$ (EI): m/z (\%): 136 [M]+ (94), 121 (62), 107 (100), 94 (49), 80 (69). HRMS (EI): $m / z$ calcd. for $\mathrm{C}_{8} \mathrm{H}_{12} \mathrm{~N}_{2}: 136.0995$ [M] $]^{+}$; Found: 136.0995.

(E)-1-Phenyl-3-styryl-1H-pyrazole (7c). ${ }^{1} \mathrm{H}$ NMR (600 MHz, $\left.\mathrm{CDCl}_{3}\right): \delta 7.92(\mathrm{~d}, J 2.5 \mathrm{~Hz}, 1 \mathrm{H}), 7.73$ (dd, J 8.5, 1.1 $\mathrm{Hz}, 2 \mathrm{H}), 7.57(\mathrm{~d}, J 7.3 \mathrm{~Hz}, 2 \mathrm{H}), 7.55(\mathrm{~d}, J 7.4 \mathrm{~Hz}, 2 \mathrm{H}), 7.48(\mathrm{dd}, J 8.5,7.5 \mathrm{~Hz}, 2 \mathrm{H}), 7.39(\mathrm{t}, J 7.6 \mathrm{~Hz}, 2 \mathrm{H}), 7.30(\mathrm{t}, J$ $7.3 \mathrm{~Hz}, 1 \mathrm{H}), 7.29(\mathrm{t}, J 7.5 \mathrm{~Hz}, 1 \mathrm{H}), 7.26(\mathrm{~d}, J 16.6 \mathrm{~Hz}, 1 \mathrm{H}), 7.20(\mathrm{~d}, J 16.6 \mathrm{~Hz}, 1 \mathrm{H}), 6.71(\mathrm{~d}, J 2.5 \mathrm{~Hz}, 1 \mathrm{H}) .{ }^{13} \mathrm{C} \mathrm{NMR}$ $\left(150 \mathrm{MHz}, \mathrm{CDCl}_{3}\right): \delta 152.4(\mathrm{C}), 140.2(\mathrm{C}), 137.2(\mathrm{C}), 130.9(\mathrm{CH}), 129.6(2 \mathrm{CH}), 128.8(2 \mathrm{CH}), 128.0(\mathrm{CH}), 127.9$ (CH), $126.7(2 \mathrm{CH}), 126.5(\mathrm{CH}, \mathrm{C} 13), 120.6(\mathrm{CH}), 119.1(2 \mathrm{CH}), 105.1(\mathrm{CH})$. IR (ATR): $v_{\max } 3047,3000,1598,1515$, 1499, 1381, 1256, 1052, 963, 946, 761, 747, $688 \mathrm{~cm}^{-1} . \mathrm{MS}$ (EI): $\mathrm{m} / \mathrm{z}$ (\%): $246[\mathrm{M}]^{+}$(84), 245 (100), 169 (39), 168 (31), 158 (39). HRMS (EI): $m / z$ calcd. for $\mathrm{C}_{17} \mathrm{H}_{14} \mathrm{~N}_{2}[\mathrm{M}]^{+}: 246.1152$; Found: 246.1152.

3-((1R,5S)-6,6-Dimethylbicyclo[3.1.1] hept-2-en-2-yl)-1-phenyl-1H-pyrazole (7d). ${ }^{1} \mathrm{H} \mathrm{NMR}(600 \mathrm{MHz}, \mathrm{CDCl} 3): \delta$ 7.86 (d, J $2.5 \mathrm{~Hz}, 1 \mathrm{H}$ ), 7.70 (dd, J 8.7, $1.2 \mathrm{~Hz}, 2 \mathrm{H}$ ), 7.44 (dd, J 8.5, 7.4 Hz, 2H), 7.26 (tt, J 7.5, 1.1 Hz, $2 \mathrm{H}$ ), 6.55 (d, J $2.5 \mathrm{~Hz}, 1 \mathrm{H}), 6.19(\mathrm{tt}, J 3.3,1.5 \mathrm{~Hz}, 1 \mathrm{H}), 3.12(\mathrm{td}, J 5.7,1.4 \mathrm{~Hz}, 1 \mathrm{H}), 2.55(\mathrm{dt}, J 8.8,5.7 \mathrm{~Hz}, 1 \mathrm{H}), 2.51(\mathrm{dt}, J 18.4$, $3.2 \mathrm{~Hz}, 1 \mathrm{H}), 2.44(\mathrm{dt}, J 18.4,3.0 \mathrm{~Hz}, 1 \mathrm{H}), 2.22$ (septd, J 2.9, $1.2 \mathrm{~Hz}, 1 \mathrm{H}), 1.41(\mathrm{~s}, 3 \mathrm{H}), 1.34(\mathrm{~d}, J 8.9 \mathrm{~Hz}, 1 \mathrm{H}), 0.93$ (s, 3H). ${ }^{13} \mathrm{C} \mathrm{NMR}\left(150 \mathrm{MHz}, \mathrm{CDCl}_{3}\right): \delta 153.7$ (C), 140.9 (C), $140.4(\mathrm{C}), 129.4(2 \mathrm{CH}), 127.7(\mathrm{CH}), 126.0(\mathrm{CH}), 120.7$ $(\mathrm{CH}), 119.0(2 \mathrm{CH}), 104.1(\mathrm{CH}), 43.0(\mathrm{CH}), 41.0(\mathrm{CH}), 38.0(\mathrm{C}), 32.0\left(\mathrm{CH}_{2}\right), 31.6\left(\mathrm{CH}_{2}\right), 26.4\left(\mathrm{CH}_{3}\right), 21.1\left(\mathrm{CH}_{3}\right) . \mathrm{IR}$ (ATR): $v_{\max } 2931,2913,1599,1517,1501,1461,1365,1315,1254,1220,1199,1176,1155,1140,1121,1081$, 1046, 1029, 946, 927, 902, 885, 807, 751, 689, $667 \mathrm{~cm}^{-1} . \mathrm{MS}$ (EI): m/z (\%): 264 [M] (92), 249 (78), 221 (100), 182 (40), 105 (37). HRMS (EI): $m / z$ calcd. for $\mathrm{C}_{18} \mathrm{H}_{20} \mathrm{~N}_{2}$ [M] $]^{+}: 264.1621$; Found: 264.1621. 
(S)-1-Phenyl-3-[4-(prop-1-en-2-yl)cyclohex-1-en-1-yl]-1H-pyrazole (7e). ${ }^{1} \mathrm{H}$ NMR $\left(600 \mathrm{MHz}, \mathrm{CDCl}_{3}\right): \delta 7.86(\mathrm{~d}, J$ $2.3 \mathrm{~Hz}, 1 \mathrm{H}), 7.70(\mathrm{dd}, J$ 8.5, $1.1 \mathrm{~Hz}, 2 \mathrm{H}), 7.44(\mathrm{dd}, J 8.4,7.4 \mathrm{~Hz}, 2 \mathrm{H}), 7.26(\mathrm{tt}, J 7.5,1.1 \mathrm{~Hz}, 1 \mathrm{H}), 6.53(\mathrm{~d}, J 2.4 \mathrm{~Hz}$, $1 \mathrm{H}), 6.43(\mathrm{dtd}, J 5.2,2.4,0.9 \mathrm{~Hz}, 1 \mathrm{H}), 4.80-4.78(\mathrm{~m}, 2 \mathrm{H}), 2.84-2.79(\mathrm{~m}, 1 \mathrm{H}), 2.56-2.49(\mathrm{~m}, 1 \mathrm{H}), 2.40-2.35(\mathrm{~m}$, $1 \mathrm{H}), 2.32-2.27(\mathrm{~m}, 1 \mathrm{H}), 2.17$ (dddt, J 17.6, 10.5, 3.9, $2.6 \mathrm{~Hz}, 1 \mathrm{H}), 2.02-1.98(\mathrm{~m}, 1 \mathrm{H}), 1.81(\mathrm{~s}, 3 \mathrm{H}), 1.64(\mathrm{dtd}, J$ 12.7, 11.5, $5.3 \mathrm{~Hz}, 1 \mathrm{H}) .{ }^{13} \mathrm{C} \mathrm{NMR}\left(150 \mathrm{MHz}, \mathrm{CDCl}_{3}\right): \delta 154.7$ (C), 150.0 (C), 140.4 (C), 130.4 (C), 129.5 (2CH), $127.4(\mathrm{CH}), 126.1(\mathrm{CH}), 124.8(\mathrm{CH}), 118.9(2 \mathrm{CH}), 108.9\left(\mathrm{CH}_{2}\right), 104.0(\mathrm{CH}), 41.1(\mathrm{CH}), 31.1\left(\mathrm{CH}_{2}\right), 27.7\left(\mathrm{CH}_{2}\right), 26.5$ $\left(\mathrm{CH}_{2}\right), 21.0\left(\mathrm{CH}_{3}\right)$. IR (ATR): $v_{\max }$ 3069, 2964, 2917, 1599, 1515, 1501, 1451, 1434, 1401, 1372, 1346, 1329, $1294,1254,1236,1093,1039,948,886,830,750,688 \mathrm{~cm}^{-1} . \mathrm{MS}$ (EI): $\mathrm{m} / z$ (\%): 264 [M] $^{+}(89), 249$ (33), 223 (87), 195 (100), 182 (82), 169 (45). HRMS (EI): $\mathrm{m} / z$ calcd. for: $\mathrm{C}_{18} \mathrm{H}_{20} \mathrm{~N}_{2}$ [M] ${ }^{+}: 264.1621$; Found: 264.1621.

(E)-1-Phenyl-3-(prop-1-en-1-yl)-1H-pyrazole (7g). ${ }^{1} \mathrm{H}$ NMR (400 MHz, CDCl 3 ): $\delta 7.84$ (d, J 2.5 Hz, $1 \mathrm{H}$ ), 7.67 (dd, J 8.5, $1.1 \mathrm{~Hz}, 2 \mathrm{H}), 7.44(\mathrm{dd}, J$ 8.3, $7.5 \mathrm{~Hz}, 2 \mathrm{H}), 7.26(\mathrm{tt}, J 7.4,1.1 \mathrm{~Hz}, 2 \mathrm{H}), 6.53(\mathrm{dq}, J$ 16.0, $1.5 \mathrm{~Hz}, 1 \mathrm{H}), 6.51(\mathrm{~d}, J 2.5$ $\mathrm{Hz}, 1 \mathrm{H}), 6.35$ (dq, J 15.9, $6.6 \mathrm{~Hz}, 1 \mathrm{H}), 1.92$ (dd, J 6.6, $1.6 \mathrm{~Hz}, 1 \mathrm{H}) .{ }^{13} \mathrm{C} \mathrm{NMR}\left(150 \mathrm{MHz}, \mathrm{CDCl}_{3}\right): \delta 152.7$ (C), 140.2 (C), $129.5(2 \mathrm{CH}), 128.6(\mathrm{CH}), 127.7(\mathrm{CH}), 126.2(\mathrm{CH}), 123.2(\mathrm{CH}), 119.0(2 \mathrm{CH}), 104.4(\mathrm{CH}), 18.6\left(\mathrm{CH}_{3}\right)$. IR (ATR): $V_{\max }$ 3048, 3013, 2962, 2931, 2912, 1598, 1518, 1500, 1457, 1386, 1369, 1257, 1048, 963, 949, 930, 750, 689 $\mathrm{cm}^{-1}$. MS (EI): m/z (\%): 184 [M] ${ }^{+}$(100), 183 (29), 77 (39). HRMS (EI): $\mathrm{m} / z$ calcd. for $\mathrm{C}_{12} \mathrm{H}_{12} \mathrm{~N}_{2}[\mathrm{M}]^{+}:$184.0995; Found: 184.0995.

(E)-3-(Pent-1-en-1-yl)-1-phenyl-1H-pyrazole (7j). ${ }^{1} \mathrm{H}$ NMR (400 MHz, $\left.\mathrm{CDCl}_{3}\right): \delta 7.84(\mathrm{~d}, J 2.5 \mathrm{~Hz}, 1 \mathrm{H}), 7.68(\mathrm{dd}, J$ 8.5, $1.3 \mathrm{~Hz}, 1 \mathrm{H}$ ), 7.44 (dd, J 8.5, $7.5 \mathrm{~Hz}, 2 \mathrm{H}), 7.26(\mathrm{tt}, J$ 7.4, $1.1 \mathrm{~Hz}, 1 \mathrm{H}), 6.53(\mathrm{dt}, J$ 16.0, $1.4 \mathrm{~Hz}, 1 \mathrm{H}), 6.52(\mathrm{~d}, J 2.5$ $\mathrm{Hz}, 1 \mathrm{H}), 6.34$ (dt, J 16.0, $6.9 \mathrm{~Hz}, 1 \mathrm{H}), 2.23$ (qd, J 7.0, 1.4 Hz, 2H), 1.53 (sext, J $7.4 \mathrm{~Hz}, 2 \mathrm{H}), 0.98(\mathrm{t}, J 7.3 \mathrm{~Hz}, 3 \mathrm{H})$. ${ }^{13} \mathrm{C} \mathrm{NMR}\left(150 \mathrm{MHz}, \mathrm{CDCl}_{3}\right): \delta 152.8(\mathrm{C}), 140.3(\mathrm{C}), 133.8(\mathrm{CH}), 129.5(2 \mathrm{CH}), 127.6(\mathrm{CH}), 126.2(\mathrm{CH}), 122.1(\mathrm{CH})$, $118.9(2 \mathrm{CH}), 104.5(\mathrm{CH}), 35.1\left(\mathrm{CH}_{2}\right), 22.4\left(\mathrm{CH}_{2}\right), 13.9\left(\mathrm{CH}_{3}\right) . \mathrm{IR}(\mathrm{ATR}): v_{\max } 3047,2958,2928,1599,1518,1501$, 1456, 1257, 1046, 965, 947, 749, $689 \mathrm{~cm}^{-1} . \mathrm{MS}$ (EI): m/z (\%): 212 [M] (100), 197 (80), 183 (38), 131 (34), 77 (31). HRMS (EI): $m / z$ calcd. for $\mathrm{C}_{14} \mathrm{H}_{16} \mathrm{~N}_{2}[\mathrm{M}]^{+}:$212.1308; Found: 212.1308.

(E)-1-Phenyl-5-styryl-1H-pyrazole (8c). ${ }^{1} \mathrm{H}$ NMR (600 MHz, CDCl $)$ : $\delta 7.69$ (d, J $\left.1.9 \mathrm{~Hz}, 1 \mathrm{H}\right), 7.53(\mathrm{~d}, J 4.3 \mathrm{~Hz}, 4 \mathrm{H})$, 7.45 (q, J $4.3 \mathrm{~Hz}, 1 \mathrm{H}), 7.43$ (d, J $7.9 \mathrm{~Hz}, 2 \mathrm{H}), 7.35$ (t, J $7.6 \mathrm{~Hz}, 2 \mathrm{H}), 7.29$ (tt, J 7.4, $1.1 \mathrm{~Hz}, 1 \mathrm{H}), 7.11(\mathrm{~d}, J 16.3 \mathrm{~Hz}$, 1H), 6.92 (d, J $16.3 \mathrm{~Hz}, 1 \mathrm{H}), 6.69$ (d, J $1.9 \mathrm{~Hz}, 1 \mathrm{H}) .{ }^{13} \mathrm{C} \mathrm{NMR}\left(150 \mathrm{MHz}, \mathrm{CDCl}_{3}\right): \delta 141.3(\mathrm{C}), 140.4(\mathrm{CH}), 139.7$ (C), $136.5(\mathrm{C}), 132.2(\mathrm{CH}), 129.3(2 \mathrm{CH}), 128.9(2 \mathrm{CH}), 128.4(\mathrm{CH}), 128.1(\mathrm{CH}), 126.7(2 \mathrm{CH}), 125.6(2 \mathrm{CH}), 115.8(\mathrm{CH})$, 104.2 (CH). IR (ATR): $v_{\max } 3046,3026,3002,1598,1515,1499,1455,1389,1328,1256,1237,1071,1052,962$,

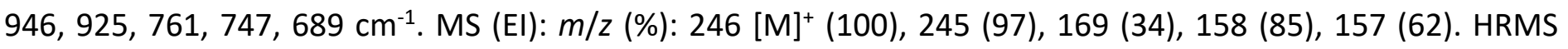
(EI): $m / z$ calcd. for $\mathrm{C}_{17} \mathrm{H}_{14} \mathrm{~N}_{2}[\mathrm{M}]^{+}:$246.1152; Found: 246.1152 .

5-((1R,5S)-6,6-Dimethylbicyclo[3.1.1] hept-2-en-2-yl)-1-phenyl-1H-pyrazole (8d). ${ }^{1} \mathrm{H} N M R(600 \mathrm{MHz} \mathrm{CDCl}): \delta$ 7.60 (d, J $1.9 \mathrm{~Hz}, 1 \mathrm{H}), 7.47$ (dd, J 8.5, $1.2 \mathrm{~Hz}, 2 \mathrm{H}$ ), 7.42 (t, J $7.9 \mathrm{~Hz}, 2 \mathrm{H}), 7.34$ (tt, J 7.3, 1.3 Hz, $1 \mathrm{H}$ ), 6.25 (d, J 1.9 $\mathrm{Hz}, 1 \mathrm{H}), 5.75(\mathrm{tt}, J 3.3,1.4 \mathrm{~Hz}, 1 \mathrm{H}), 2.40(\mathrm{dt}, J 18.6,3.1 \mathrm{~Hz}, 1 \mathrm{H}), 2.31(\mathrm{dt}, J 8.8,5.7 \mathrm{~Hz}, 1 \mathrm{H}), 2.29(\mathrm{dt}, J 18.6,3.1$, $1 \mathrm{H}), 2.11-2.08(\mathrm{~m}, 1 \mathrm{H}), 2.07(\mathrm{td}, J 5.6,1.5 \mathrm{~Hz}, 1 \mathrm{H}), 1.25(\mathrm{~d}, J 8.8 \mathrm{~Hz}, 1 \mathrm{H}), 1.18(\mathrm{~s}, 3 \mathrm{H}), 0.87(\mathrm{~s}, 3 \mathrm{H}) .{ }^{13} \mathrm{C} \mathrm{NMR}(150$ $\left.\mathrm{MHz}, \mathrm{CDCl}_{3}\right): \delta 144.1(\mathrm{C}), 141.0(\mathrm{C}), 140.1(\mathrm{CH}), 137.6(\mathrm{C}), 129.0(2 \mathrm{CH}), 127.6(\mathrm{CH}), 125.2(\mathrm{CH}), 124.9(2 \mathrm{CH})$, $106.2(\mathrm{CH}), 45.8(\mathrm{CH}), 40.2(\mathrm{CH}), 38.0(\mathrm{C}), 32.1\left(\mathrm{CH}_{2}\right), 31.9\left(\mathrm{CH}_{2}\right), 26.2\left(\mathrm{CH}_{3}\right), 20.9\left(\mathrm{CH}_{3}\right)$. IR $(\mathrm{ATR}): v_{\max } 2946$, 2933, 2915, 1597, 1499, 1468, 1448, 1383, 1366, 1349, 1263, 1219, 1117, 1070, 1055, 1021, 952, 924, 900,

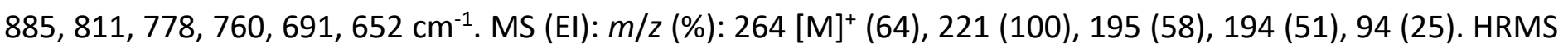
(EI): $m / z$ calcd. for: $\mathrm{C}_{18} \mathrm{H}_{20} \mathrm{~N}_{2}[\mathrm{M}]^{+}:$264.1621; Found: 264.1621.

(S)-1-Phenyl-5-[4-(prop-1-en-2-yl)cyclohex-1-en-1-yl]-1H-pyrazole (8e). ${ }^{1} \mathrm{H}$ NMR $\left(600 \mathrm{MHz}, \mathrm{CDCl}_{3}\right): \delta 7.62(\mathrm{~d}, J$ $1.9 \mathrm{~Hz}, 1 \mathrm{H}), 7.49(\mathrm{dd}, J$ 8.3, $1.0 \mathrm{~Hz}, 2 \mathrm{H}), 7.43(\mathrm{t}, J 7.9 \mathrm{~Hz}, 2 \mathrm{H}), 7.35(\mathrm{tt}, J 7.4,1.2 \mathrm{~Hz}, 1 \mathrm{H}), 6.28(\mathrm{~d}, J 1.8 \mathrm{~Hz}, 1 \mathrm{H})$, 5.87-5.85 (m, 1H), 4.79 (quint, J $1.4 \mathrm{~Hz}, 1 \mathrm{H}), 4.72-4.71(\mathrm{~m}, 1 \mathrm{H}), 2.27-2.21(\mathrm{~m}, 1 \mathrm{H}), 2.20-2.16(\mathrm{~m}, 1 \mathrm{H}), 2.15-2.10$ $(\mathrm{m}, 2 \mathrm{H}), 2.07-2.01(\mathrm{~m}, 1 \mathrm{H}), 1.82-1.78(\mathrm{~m}, 1 \mathrm{H}), 1.74(\mathrm{~s}, 3 \mathrm{H}), 1.49$ (dddd, J 12.8, 11.2, 10.1, 6.1 Hz, $1 \mathrm{H}) .{ }^{13} \mathrm{C} \mathrm{NMR}$ $\left(150 \mathrm{MHz}, \mathrm{CDCl}_{3}\right.$ ): $\delta 149.3$ (C), 145.1 (C), 140.9 (C), $139.9(\mathrm{CH}), 130.0(\mathrm{CH}), 129.1(2 \mathrm{CH}), 128.0(\mathrm{C}), 127.5(\mathrm{CH})$, 
$124.6(2 \mathrm{CH}), 109.1\left(\mathrm{CH}_{2}\right), 106.5(\mathrm{CH}), 40.3(\mathrm{CH}), 31.0\left(\mathrm{CH}_{2}\right), 28.5\left(\mathrm{CH}_{2}\right), 27.5\left(\mathrm{CH}_{2}\right), 20.9\left(\mathrm{CH}_{3}\right)$. IR $(\mathrm{ATR}): V_{\max }$ 3046, 2964, 2917, 1597, 1515, 1500, 1473, 1449, 1384, 1328, 1312, 1258, 1229, 1122, 1070, 1052, 963, 945, 925, 889, 833, 760, 690, $651 \mathrm{~cm}^{-1} . \mathrm{MS}$ (EI): m/z (\%): 264 [M]+ (77), 263 (44), 196 (33), 195 (92), 94 (100). HRMS (EI): $m / z$ calcd. for: $\mathrm{C}_{18} \mathrm{H}_{20} \mathrm{~N}_{2}[\mathrm{M}]^{+}:$: 264.1621; Found: 264.1621.

(E)-1-Phenyl-5-(prop-1-en-1-yl)-1H-pyrazole (8g). ${ }^{1} \mathrm{H}$ NMR (600 MHz, CDCl 3$): \delta 7.59$ (d, J 1.9 Hz, 1H), 7.51-7.44 $(\mathrm{m}, 4 \mathrm{H}), 7.42-7.38(\mathrm{~m}, 1 \mathrm{H}), 6.47(\mathrm{~d}, J 1.9 \mathrm{~Hz}, 1 \mathrm{H}), 6.32-6.21(\mathrm{~m}, 2 \mathrm{H}), 1.85(\mathrm{~d}, J 4.9 \mathrm{~Hz}, 3 \mathrm{H}) .{ }^{13} \mathrm{C} \mathrm{NMR}(150 \mathrm{MHz}$, $\left.\mathrm{CDCl}_{3}\right): \delta 141.5(\mathrm{C}), 140.2(\mathrm{CH}), 139.8(\mathrm{C}), 130.2(\mathrm{CH}), 129.2(2 \mathrm{CH}), 127.9(\mathrm{CH}), 125.5(2 \mathrm{CH}), 118.9(\mathrm{CH}), 103.6$ (CH), $18.7\left(\mathrm{CH}_{3}\right)$. IR (ATR): $v_{\max }$ 3033, 2963, 2933, 1596, 1499, 1452, 1391, 1070, 956, 931, 924, 910, 761, 695, 684, $648 \mathrm{~cm}^{-1}$. MS (EI): m/z (\%): 184 [M] ${ }^{+}$(100), 183 (35), 77 (33). HRMS (EI): m/z calcd. for $\mathrm{C}_{12} \mathrm{H}_{12} \mathrm{~N}_{2}: 184.0995$ $[\mathrm{M}]^{+}$; Found: 184.0995.

5-(2-Methylprop-1-en-1-yl)-1-phenyl-1H-pyrazole (8i). ${ }^{1} \mathrm{H} \mathrm{NMR}\left(600 \mathrm{MHz}, \mathrm{CDCl}_{3}\right): \delta 7.65(\mathrm{~d}, J 1.8 \mathrm{~Hz}, 1 \mathrm{H}), 7.48$ (dd, J 8.4, 1.1 Hz, 2H), 7.43 (t, J 7.6 Hz, 2H), 7.34 (tt, J 7.4, 1.2 Hz, 1H), 6.37 (d, J $1.8 \mathrm{~Hz}, 1 \mathrm{H}), 5.97$ (br s, $1 \mathrm{H}), 1.92$ (d, J $1.0 \mathrm{~Hz}, 3 \mathrm{H}), 1.86$ (d, J $1.2 \mathrm{~Hz}, 3 \mathrm{H}) .{ }^{13} \mathrm{C} \mathrm{NMR}\left(150 \mathrm{MHz}, \mathrm{CDCl}_{3}\right): \delta 140.1$ (C), $140.0(\mathrm{C}), 139.9$ (CH), $139.4(\mathrm{C})$, $129.0(2 \mathrm{CH}), 127.5(\mathrm{CH}), 125.2(2 \mathrm{CH}), 113.5(\mathrm{CH}), 106.9(\mathrm{CH}), 26.8\left(\mathrm{CH}_{3}\right), 20.1\left(\mathrm{CH}_{3}\right)$. HRMS (EI): $\mathrm{m} / \mathrm{z}$ calcd. for $\mathrm{C}_{13} \mathrm{H}_{14} \mathrm{~N}_{2}: 198.1152[\mathrm{M}]^{+}$; Found: 198.1152.

(E)-5-(Pent-1-en-1-yl)-1-phenyl-1H-pyrazole (8j). ${ }^{1} \mathrm{H}$ NMR (400 MHz, $\mathrm{CDCl}_{3}$ ): $\delta 7.60$ (d, J 1.9 Hz, 1H), 7.51-7.45 $(\mathrm{m}, 4 \mathrm{H}), 7.42-7.38(\mathrm{~m}, 1 \mathrm{H}), 6.48(\mathrm{~d}, J 1.9 \mathrm{~Hz}, 1 \mathrm{H}), 6.30-6.20(\mathrm{~m}, 2 \mathrm{H}), 2.18-2.13(\mathrm{~m}, 2 \mathrm{H}), 1.47$ (sext, J 7.3 Hz, 2H), $0.94(\mathrm{t}, J 7.4 \mathrm{~Hz}, 3 \mathrm{H}) .{ }^{13} \mathrm{C} \mathrm{NMR}\left(150 \mathrm{MHz}, \mathrm{CDCl}_{3}\right): \delta 141.5(\mathrm{C}), 140.2(\mathrm{CH}), 139.8(\mathrm{C}), 135.3(\mathrm{CH}), 129.2(2 \mathrm{CH})$, $127.8(\mathrm{CH}), 125.5(2 \mathrm{CH}), 117.8(\mathrm{CH}), 103.6(\mathrm{CH}), 35.2\left(\mathrm{CH}_{2}\right), 22.3\left(\mathrm{CH}_{2}\right), 13.8\left(\mathrm{CH}_{3}\right)$. IR (ATR): $v_{\max } 3034,2958$, 2929, 1598, 1500, 1448, 1391, 961, 931, 924, 761, 695, 685, $649 \mathrm{~cm}^{-1} . \mathrm{MS}$ (EI): $\mathrm{m} / z$ (\%): $212[\mathrm{M}]^{+}$(70), 208 (30), 183 (70), 169 (100), 131 (33), 77 (45). HRMS (EI): m/z calcd. for $\mathrm{C}_{14} \mathrm{H}_{16} \mathrm{~N}_{2}$ [M] ${ }^{+}:$212.1308; Found: 212.1308.

\section{Acknowledgements}

This work was supported by the German Research Foundation DFG (No. 265182801).

\section{Supplementary Material}

See the Supporting Information for full experimental data and copies of ${ }^{1} \mathrm{H}$ and ${ }^{13} \mathrm{C}$ NMR spectra.

\section{References}

1. Küçükgüzel, Ş. G.; Şenkardeş, S. Eur. J. Med. Chem. 2015, 97, 786-815. https://doi.org/10.1016/i.ejmech.2014.11.059

2. Karrouchi, K.; Radi, S.; Ramli, Y.; Taoufik, J.; Mabkhot, Y. N.; Al-aizari, F. A.; Ansar, M. Molecules 2018, 23, 134.

https://doi.org/10.3390/molecules23010134

3. Fustero, S.; Sánchez-Roselló, M.; Barrio, P.; Simón-Fuentes, A. Chem. Rev. 2011, 111, 6984-7034. https://doi.org/10.1021/cr2000459

4. Stanovnik, B.; Svete, J. In Pyrazoles; Neier, R., Ed.; Georg Thieme: Stuttgart, Germany, 2002; Science of Synthesis, Houben-Weyl, Methods of Organic Transformations Vol. 12, p 15. 
5. Heller, S. T.; Natarajan, S. R. Org. Lett., 2006, 8, 2675-2678. https://doi.org/10.1021/ol060570p

6. Kumar, S. V.; Yadav, S. K.; Raghava, B.; Saraiah, B.; Ila, H.; Rangappa, K. S.; Hasra, A. J. Org. Chem. 2013, 78, 4960-4973.

https://doi.org/10.1021/jo400599e

7. Harigae, R.; Moriyama, K.; Togo, H. J. Org. Chem. 2014, 79, 2049-2058.

https://doi.org/10.1021/jo4027116

8. Zhang, X.; Kang, J.; Niu, P.; Wu, J.; Yu, W.; Chang, J. J. Org. Chem. 2014, 79, 10170-10178. https://doi.org/10.1021/jo501844x

9. Hu, J.; Chen, S.; Sun, Y.; Yang, J.; Rao, Y. Org. Lett. 2012, 14, 5030-5033. https://doi.org/10.1021/ol3022353

10. Schmitt, D. C.; Taylor, A. P.; Flick, A. C.; Kyne, R. E. Org. Lett. 2016, 18, 4206-4209. https://doi.org/10.1021/acs.orglett.6b01867

11. Ding, Y.; Zhang, T. Chen, Q.-Y.; Zhu, C. Org. Lett. 2015, 17, 1405-1408.

12. Haak, E. Eur. J. Org. Chem. 2007, 2815-2824. https://doi.org/10.1002/ejoc.200700064

13. Thies, N.; Hrib, C. G.; Haak, E. Chem. Eur. J. 2012, 18, 6302-6308. https://doi.org/10.1002/chem.201200188

14. Jonek, A.; Berger, S.; Haak, E. Chem. Eur. J. 2012, 18, 15504-15511. https://doi.org/10.1002/chem.201202414

15. Berger, S.; Haak, E. Tetrahedron Lett. 2010, 51, 6630-6634. https://doi.org/10.1016/i.tetlet.2010.10.053

16. Kaufmann, J.; Jäckel, E.; Haak, E. Angew. Chem. 2018, 130, 6010-6014; Angew. Chem. Int. Ed. 2018, 57, 5908-5911.

https://doi.org/10.1002/anie.201801846

17. Thies, N.; Gerlach, M.; Haak, E. Eur. J. Org. Chem. 2013, 7354-7365. https://doi.org/10.1002/ejoc.201300803

18. Thies, N.; Haak, E. Angew. Chem. 2015, 127, 4170-4174; Angew. Chem. Int. Ed. 2015, 54, 4097-4101. https://doi.org/10.1002/anie.201412207

19. Thies, N.; Stürminger, M.; Haak, E. Synlett 2017, 28, 701-704.

20. Jäckel, E.; Kaufmann, J.; Haak, E. Synthesis 2018, 50, 742-752. https://doi.org/10.1055/s-0036-1591735

21. Fukumoto, Y.; Tamura, Y.; Iyori, Y.; Chatani, N. J. Org. Chem. 2016, 81, 3161-3167. https://doi.org/10.1021/acs.joc.6b00116

22. Levanova, E. P; Grabel'nykh, V. A.; Volkova, K. A.; Russavskaya, N. V.; Klyba, L. V.; Albanov, A. I.; Korchevin, N. A. Chem. Heterocycl. Compd. 2009, 45, 1009-1010. https://doi.org/10.1007/s10593-009-0379-3

23. Pleier, A. K.; Glas, H.; Grosche, M.; Sirsch, P.; Thiel, W. R. Synthesis 2001, 55-62. https://doi.org/10.1055/s-2001-9761

24. Ma, D.-Y.; Wang, D.-X.; Pan, J.; Huang, Z.-T.; Wang, M.-X. J. Org. Chem. 2008, 73, 4087-4091. https://doi.org/10.1021/jo800074k

25. Yamasaki, S.; Kanai, M.; Shibasaki, M. J. Am. Chem. Soc. 2001, 123, 1256-1257. https://doi.org/10.1021/ja005794w

26. Kisanga, P.; McLeod, D.; D’Sa, B.; Verkade, J. J. Org. Chem. 1999, 64, 3090-3094. 
https://doi.org/10.1021/jo981858y

27. Majerić Elenkov, M.; Hauer, B.; Janssena, D. B. Adv. Synth. Catal. 2006, 348, 579-585.

https://doi.org/10.1002/adsc.200505333

28. Jinzaki, T.; Arakawa, M.; Kinoshita, H.; Ichikawa, J.; Miura, K. Org. Lett. 2013, 15, 3750-3753. https://doi.org/10.1021/ol401663u

29. Pori, M.; Galletti, P.; Soldati, R.; Calzà, L.; Mangano, C.; Giacomini, D. Eur. J. Med. Chem. 2013, 70, $857-863$. https://doi.org/10.1016/j.ejmech.2013.09.057

30. Cristau, H.-J.; Cellier, P. P.; Spindler, J.-F.; Taillefer, M. Eur. J. Org. Chem. 2004, 695-709. https://doi.org/10.1002/ejoc.200300709

31. Browne, D. L.; Taylor, J. B.; Plant, A.; Harrity, J. P. A. J. Org. Chem. 2010, 75, 984-987. https://doi.org/10.1021/j0902514v

32. Begtrup, M.; Veds $\varnothing$, P.; Cabildo, P.; Claramunt, R. M.; Elguero, J.; Meuter-Mans, W. Magn. Reson. Chem. 1992, 30, 455-459.

https://doi.org/10.1002/mrc.1260300518 